\title{
Biochemical Studies in a Patient with Subacute Neuropathic Gaucher Disease without Visceral Glucosylceramide Storage
}

\author{
DAVID A. WENGER, ${ }^{(23)}$ SANDRA ROTH, TOORU KUDOH, WARREN D. GROVER, \\ SAMUEL H. TUCKER, EDWARD M. KAYE, AND M. DAVID ULLMAN \\ Department of Pediatrics, Box C-233, University of Colorado School of Medicine, Denver, Colorado [D.A.W., S.R., \\ T.K.]; Section of Child Neurology, St. Christopher's Hospital for Children, Temple University Medical Center, \\ Philadelphia, Pennsylvania [W.D.G.]; Section of Pediatric Neurology, Children's Hospital, Philadelphia, Pennsylvania \\ [S.H.T.]; and GRECC, Bedford VA Medical Center, Bedford, Massachusetts, USA [E.M.K., M.D.U.]
}

\begin{abstract}
Summary
Autopsy samples were obtained from a 12.5-year-old girl who died with a neurologic disorder consisting of myoclonus, myoclonic epilepsy, spasticity, strabismus, and mild mental retardation but no hepatosplenomegaly. Studies in leukocytes, cultured skin fibroblasts, brain, liver, and spleen of this patient revealed glucosylceramide $\beta$-glucosidase (EC 3.2.1.45, glucocerebrosidase) activity about $10 \%$ of controls, and well in the range found in samples from Gaucher disease patients. Extraction of the lipids from liver and spleen with chloroform-methanol (2:1) did not show accumulation of glucosylceramide or other lipid. Examination of the lipids in brain by high performance liquid chomatography revealed the presence of glucosylceramide, which is not found in brain samples from controls. Pathologic examination of the liver and spleen revealed no evidence of Gaucher disease. The brain showed many degenerative lesions and loss of neurons. There was no complementation of glucocerebrosidase activity when the cells from this patient were hybridized with cells from patients with Type 1 or Type 2 Gaucher disease. The reason for the lack of glucosylceramide storage in the liver and spleen has not been determined.
\end{abstract}

\section{Abbreviations}

4M- $\beta$-gal, 4-methylumbelliferyl- $\beta$-D-galactopyranoside $4 \mathrm{Mu}-\beta$-glc, 4-methylumbelliferyl- $\beta$-D-glucopyranoside GD, Gaucher disease glc-cer, glucosylceramide GM3- $N$-acetylneuraminyl-galactosyl-glucosyl-ceramide

Gaucher disease is a group of autosomal recessive genetic diseases resulting from the deficiency of glucosylceramide $\beta$-glucosidase (EC 3.2.1.45, glucocerebrosidase) activity (1). Three clinical forms are commonly recognized, although significant differences in severity are found within each type. The non-neuropathic, Type 1, patients present initially with splenomegaly and thrombocytopenia anytime from the first few years of life until the ninth decade. Variable degrees of bone, liver, and lung involvement can be found in these patients. The infantile, acute neuropathic, Type 2 , patients usually present with massive hepatosplenomegaly and developmental delay within the first few months of life. This laboratory has diagnosed such a patient $6 \mathrm{~h}$ after birth. Continued progression of the neurologic symptoms, including spasticity, cranial nerve dysfunction and mental retardation result in death by $1-2$ years of age. The juvenile, subacute neuropathic, Type 3, patients are less well defined clinically, although, symptoms related to splenomegaly, anemia, and neurologic deterioration occur during the first decade of life and result in death by the second or third decade. The reason for this clinical heterogeneity is not known (20).
In 1978 we described two second cousins with different clinical types of Gaucher disease (5). Patient 1 had an acute neuropathic form of Gaucher disease with massive hepatosplenomegaly and neurologic symptoms including bilateral sixth nerve paresis, recurrent stridor, seizures, myoclonus and spasticity. When she died at 32 months of age from pneumonia, organs were received and analysis revealed a severe deficiency of glucocerebrosidase activity in all organs (confirming the findings in leukocytes and cultured skin fibroblasts) and storage of glucosylceramide in liver and spleen. Patient 2 was tested for Gaucher disease although she did not have typical symptoms associated with Gaucher disease. She had a severe movement disorder and mild mental retardation but no hepatosplenomegaly. Diagnosis of Gaucher disease was based on the severe deficiency of glucocerebrosidase activity in leukocytes and cultured skin fibroblasts. When Patient 2 died at 12.5 years of age, frozen tissues were received after autopsy. The studies described here confirm the deficiency of glucocerebrosidase activity in liver, spleen, and brain, but demonstrate that there was no storage of glucosylceramide in spleen or liver; however, there was an increase of this lipid in brain. This is the first case of a patient with a deficiency of glucocerebrosidase activity in five different tissue samples and no storage of glucosylceramide in liver or spleen, only in brain. This may be a form of Gaucher disease that has only neurologic symptoms and no visceral involvement. A preliminary report of this research has been presented (19).

\section{MATERIALS AND METHODS}

Leukocytes and fibroblast cultures were prepared as described previously $(16,17)$. Pellets of cells were frozen before assay except in some cases where they were assayed before freezing to rule out the existence of an enzyme unstable to freezing. All pellets were homogenized in distilled water in small Duall homogenizers (Kontes, Vineland, NJ). The total homogenates were used for all assays. Frozen samples of liver, spleen, and brain from controls and Gaucher disease patients were thawed and homogenized in 10 volumes of sodium phosphate buffer $(0.01 \mathrm{M}, \mathrm{pH} 7.0)$ containing $0.1 \mathrm{M}$ sodium chloride and $0.5 \%$ Triton $\mathrm{X}-100$. An aliquot was saved and the remainder was centrifuged at $50,000 \times g$ for 30 $\mathrm{min}$. The supernatant was removed and the pellet resuspended in the starting buffer. Protein concentrations were determined by the method of Lowry et al. (7) using bovine serum albumin as standard. All samples were collected with informed consent.

Cell hybridization experiments were carried out by the method of Davidson and Gerald (2). The cells were trypsinized, suspended in media and counted. Equal numbers of cells to be hybridized were inoculated into small flasks $(20,000$ cells/T-25 flask) and allowed to attach for $24 \mathrm{~h}$. The cells were treated with polyethylene glycol (Research Poducts International Corp., Elk Grove Village, IL) for $1 \mathrm{~min}$ followed by the addition of fresh media. After $48 \mathrm{~h}$ 
the cells were harvested and assayed immediately without freezing.

Beta-glucosidase activity was measured with synthetic substrate, 4-methylumbelliferyl- $\beta$-D-glucopyranoside (4MU- $\beta$-glc) (Sigma, St. Louis, MO) and natural substrate, $\left[{ }^{14} \mathrm{C}\right]$-glucose labeled glucosylceramide (glc-cer). The $\left[{ }^{14} \mathrm{C}\right]$-glucose labeled glc-cer was prepared according to the method of Stoffyn et al. (14) from natural ceramide produced from bovine brain sphingomyelin by the action of phospholipase $\mathrm{C}$. The assays for $\beta$-glucosidase were carried out in the presence of sodium taurocholate and oleic acid or Triton $\mathrm{X}-100$ as described previously (16). Other enzyme activities were assayed with $4 \mathrm{MU}$ substrates or radiolabeled natural substrates as described by this laboratory $(16,20)$.

The lipids in spleen and liver were extracted with 10 volumes of chloroform:methanol $(2: 1) \mathrm{v} / \mathrm{v})$ and, after filtration through glass wool, partitioned into the lower phase by the addition of one-fifth volume of distilled water. Aliquots were spotted on silica gel thin layer chromatography plates (Merck AG, Darmstadt, West Germany) and chromatographed in chloroform:methanol:water $(60: 35: 8 \mathrm{v} / \mathrm{v} / \mathrm{v})$. To visualize the glycolipids, the plates were sprayed with orcinol reagent followed by heating at $110^{\circ} \mathrm{C}$ for $10 \mathrm{~min}$. Additional aliquots were chromatographed and visualized with iodine vapor followed by scraping the glc-cer region from the plate. The lipid was eluted with chloroform:methanol:water mixtures in a glass wool-stoppered pasteur pipette. After drying the solution with nitrogen, the glc-cer concentration was measured using the anthrone method for sugar determination (13) and pure glc-cer as standard. Extractions were run in triplicate and the results are expressed as $\mathrm{mg}$ glc-cer per $\mathrm{g}$ wet weight. Samples of brain were homogenized in distilled water and an aliquot was removed for protein determination. After lyophilization the lipids were extracted with chloroform:methanol $(2: 1 \mathrm{v} / \mathrm{v})$, and after addition of one-fifth volume of distilled water and mixing the lower phase was isolated. Cerebrosides were partially purified on a silicic acid column with a $98 \%$ recovery. After drying, the samples were perbenzoylated and subjected to high performance liquid chromatography using a method only slightly modified from that of Ullman and McCluer (15).

\section{RESULTS}

Studies on leukocytes and cultured skin fibroblasts from the two second cousins demonstrated a deficiency of $\beta$-glucosidase activity using both natural and synthetic substrates (Table 1). All

Table 1. Beta-glucosidase activity in leukocytes and cultured skin fibroblasts ${ }^{1}$

\begin{tabular}{lll}
\hline \multicolumn{1}{c}{ Subjects } & $\begin{array}{c}\text { glc-cer } \\
\text { (nmole } / \mathrm{mg} \text { pro- } \\
\text { tein } / \mathrm{h})\end{array}$ & $\begin{array}{c}4 \mathrm{MU}-\beta \text {-glc } \\
(\mathrm{nmole} / \mathrm{mg} \text { pro- } \\
\text { tein } / \mathrm{h})\end{array}$ \\
\hline $\begin{array}{l}\text { Leukocytes } \\
\text { Second cousin of this case }\end{array}$ & 1.27 & 0.99 \\
$\quad$ This case & 1.33 & 0.75 \\
Gaucher disease & $1.65(9)^{2}$ & $1.15(12)^{2}$ \\
$\quad$ Mean & 0.97 & 0.26 \\
$\quad$ S.D. & $10.6(20)^{2}$ & $8.49(82)^{2}$ \\
Controls & 3.54 & 1.60 \\
$\quad$ Mean & & \\
$\quad$ S.D. & 33.1 & 32.7 \\
Fibroblast cultures & 48.1 & 34.2 \\
Second cousin of this case & & \\
This case & $28.2(11)^{2}$ & $26.0(10)^{2}$ \\
Gaucher disease & 11.3 & 11.8 \\
$\quad$ Mean & & \\
S.D. & $410(35)^{2}$ & $310(25)^{2}$ \\
Controls & 142 & 159 \\
$\quad$ Mean & & \\
S.D. &
\end{tabular}

'Abbreviations, see "Abbreviations."

${ }^{2} n$, number. other enzymes checked were in the normal range. When the younger child died at 32 months, liver and spleen obtained at autopsy revealed excessive storage of glucosylceramide and severe deficiency of $\beta$-glucosidase activity as expected from the clinical findings and the enzymatic studies in leukocytes and cultured skin fibroblasts. Gaucher cells were found in the spleen, liver, lymph nodes, bone marrow, lungs, brain and ganglion cells of the submucosal myenteric plexes of the small and large intestine. The older child died at 12.5 years of age from pneumonia. Liver, spleen, and brain were obtained at autopsy. Samples were prepared for pathology and the remainder were frozen until used for enzymatic studies and lipid analysis. Microscopic examination of the brain and spinal cord revealed many degenerative lesions. The spinal cord showed a loss of anterior horn cells, the neuropile had a loose appearance, and fiber-forming astrocytes were prominent. The cerebellar parenchyma was severely affected. Extensive Purkinje cell loss was associated with a considerable proliferation of Bergmann's astrocytes. The few remaining Purkinje cells showed acute eosinophilic necrosis. The dentate nucleus lost most of its complement of neurons and contained a massive amount of astrocytic proliferation. Sections of the pons showed only mild subependymal gliosis. Sections of the cerebral cortex displayed only a small degree of irregular neuronal loss. Examination of the spleen, bone marrow, liver, and lymph nodes did not reveal the presence of Gaucher cells.

Extraction of the liver and spleen with chloroform:methanol (2:1) resulted in a fraction rich in lipids. Thin layer chromatography revealed a normal pattern with no storage of glc-cer (Fig. 1). Quantitative studies demonstrated that glc-cer was present at the same level found in control tissues (Table 2). In addition, the pattern of phospholipids looked normal on thin layer chromatography; however, thin layer chromatography of the non-polar lipids in petroleum ether:diethylether:acetic acid (70:30:1 v/v/v) demonstrated an increase in free fatty acid in the liver but not the spleen of this older patient.

Two samples of brain from this patient were examined by high performance liquid chromatography. In both samples there was a significant concentration of glucosylceramide $(0.27$ and 0.28 nmole/mg protein). This compares to levels of 0.48 and 1.26 $\mathrm{nmole} / \mathrm{mg}$ protein in brain samples from two patients who died with acute neuropathic Gaucher disease and undetectable levels in control human brains.

The initial diagnosis of Gaucher disease was made on leukocytes and cultured skin fibroblasts, which demonstrated the $\beta$-glucosidase deficiency (Table 1). Clinically, the child had neurologic symptoms identical to some patients with juvenile Gaucher disease. Hepatosplenomegaly was not evident. To be certain that the $\beta$-glucosidase deficiency was not confined to leukocytes and cultured skin fibroblasts, samples of brain, liver and spleen were homogenized and centrifuged to provide samples for enzymatic studies. We tested three fractions: the total homogenate, the supernatant fraction, and the pellet obtained after centrifugation. The results for the total homogenate and supernatant from brain clearly demonstrate the severe deficiency of $\beta$-glucosidase activity using natural and synthetic substrates (Table 3 ). The values are in the range found in brains from three other patients with Gaucher disease. As shown in Table 3, the spleen and liver from this patient also had $\beta$-glucosidase levels within the range found in these organs from other Gaucher disease patients who showed storage of glucosylceramide. Similar results were found in the pellet extract (data not shown for reasons of space). When various enzyme fractions were mixed with the same fractions from a control and assayed, only the theoretical activities were obtained. This tends to rule out the presence of an excessive amount of an inhibiting compound in the organs and cultured cells of this patient. All other enzymes checked were normal except $\beta$-xylosidase assayed in the presence of sodium taurocholate. This was low, as we have found in other cases of Gaucher disease (20).

The reason for the low level of $\beta$-glucosidase activity in all tissue samples and absence of visceral storage of glucosylceramide remains elusive. To determine if cell hybridization with cultured 


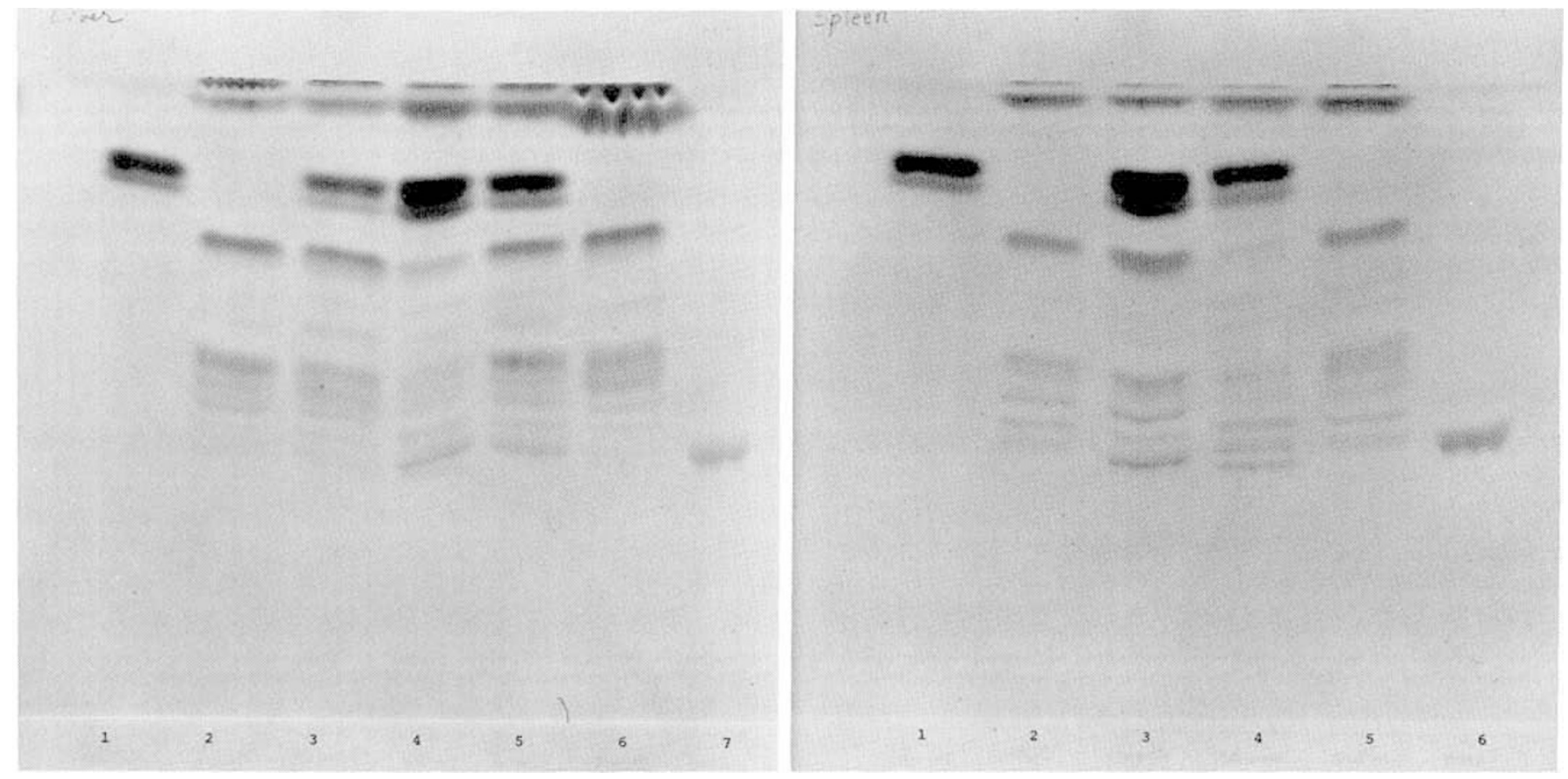

Fig. 1. Thin layer chromatography of the lipid extracts from liver (left hand panel) and spleen (right hand panel) from controls and patients. Lipids were extracted and chromatographed as described in the text. Left hand panel: Lane 1, glc-cer standard; Lane 2, control; Lane 3, Type 1 Gaucher disease; Lane 4, Type 2 Gaucher disease; Lane 5, second cousin of this patient; Lane 6, patient described in this manuscript; and Lane 7, GM3 ganglioside. Right hand panel: Lane 1, glc-cer standard; Lane 2, control; Lane 3, Type 1 Gaucher disease; Lane 4, Type 2 Gaucher disease; Lane 5, patient described in this manuscript; and Lane 6, GM3 ganglioside. See "Abbreviations" for definitions.

Table 2. Glucosylceramide concentration in liver and spleen

\begin{tabular}{lcc}
\hline \multicolumn{1}{c}{ Subjects } & $\begin{array}{c}\text { Liver } \\
(\mathrm{mg} / \mathrm{g} \text { wet } \\
\text { weight }\end{array}$ & $\begin{array}{c}\text { Spleen } \\
(\mathrm{mg} / \mathrm{g} \text { wet } \\
\text { weight })\end{array}$ \\
\hline Infantile Gaucher disease & 14.6 & 8.5 \\
Adult Gaucher disease \#1 & 5.2 & $\mathrm{ND}$ \\
Adult Gaucher disease \#2 & $\mathrm{ND}^{1}$ & 16.5 \\
Second cousin of this case & 9.1 & $\mathrm{ND}$ \\
This case & 0.6 & 0.2 \\
Controls $(n=5)$ & & \\
Mean & 1.0 & 0.9 \\
Range & $0.2-1.9$ & $0.7-0.9$ \\
\hline
\end{tabular}

${ }^{1} \mathrm{ND}$, not determined.

cells from patients with either adult or infantile Gaucher disease could result in complementation of $\beta$-glucosidase activity, fusions between different cell lines were induced with polyethylene glycol. As shown in Table 4, only theoretical values consistent with a one to one mixture of cell lines were obtained.

\section{DISCUSSION}

In this manuscript we describe a patient with a severe neurologic disease who has $\beta$-glucosidase levels consistent with a diagnosis of Gaucher disease. She is the paternal second cousin of a child who died at 32 months with a more acute neuropathic form of this disease (5). The clinical and EEG findings in the older patient, beginning as ataxia at 2.5 years and progressing to spasticity, myoclonus, and myoclonic epilepsy with mild mental retardation over the next 10 years, are similar to those reported in other patients with juvenile Gaucher disease (see references 10 and 11 for a review of the neurologic features of some Gaucher patients). The patient of Maloney and Cumings (8) had many features in common with our patient including seizures, myoclonus, ataxia, spasticity, and progressive mental retardation. Although the liver and spleen were not palpable in their patient, the spleen was found to be enlarged 3-fold at autopsy and histologic examination revealed numerous, closely packed, large cells with foamy cytoplasm and a small, often eccentric, nucleus. The liver had no evidence of lipid storage in the parechyma and only a few cells resembling foamy histocytes in the sinusoids. They found increased levels of glc-cer and phospholipids in the liver, spleen, and kidney. A large number of patients with juvenile Gaucher disease have been described in an area of Sweden $(3,6)$. A wide range of clinical symptoms were found, even among siblings; however, all of these patients had splenomegaly and other hematologic signs indicative of hypersplenism.

Parents of the younger child and the father of the older child had $\beta$-glucosidase values in leukocytes consistent with the carrier status of Gaucher disease (5). But the mother of the child described herein had higher $\beta$-glucosidase activities in her leukocytes and fibroblasts than those found in typical carriers of Gaucher disease. The mother could have a mutation in this enzyme not obvious by the usual in vitro tests which require sodium taurocholate for stimulation of glucocerebrosidase activity. When the leukocytes were assayed using a new method, involving the use of an activator protein in place of bile salt (21), the father of this child had half normal activity and the mother had low normal activity.

Examination of the brain, liver and spleen from this child revealed $\beta$-glucosidase levels in the range found in organs of other patients who died with Gaucher disease. These assays were done using both natural and synthetic substrates. The patient also had a pronounced deficiency of $\beta$-xylosidase activity when assayed with the 4MU substrate in the presence of sodium taurocholate and oleic acid (data not presented). This is consistent with our findings in other patients with Gaucher disease, including the second cousin of this patient (20). All other lysosomal enzymes assayed were in the control range. Some assays were carried out using the activator protein purified from Gaucher disease spleen in place of bile salts (21). Again, this patient had enzyme values in cultured skin fibroblasts, brain and spleen consistent with a severe deficiency of $\beta$-glucosidase activity (8-15\% of controls) using natural and synthetic substrates. 
Table 3. Hydrolase activities [nmole/mg protein $/ h$ ] in brain, liver, and spleen ${ }^{1}$

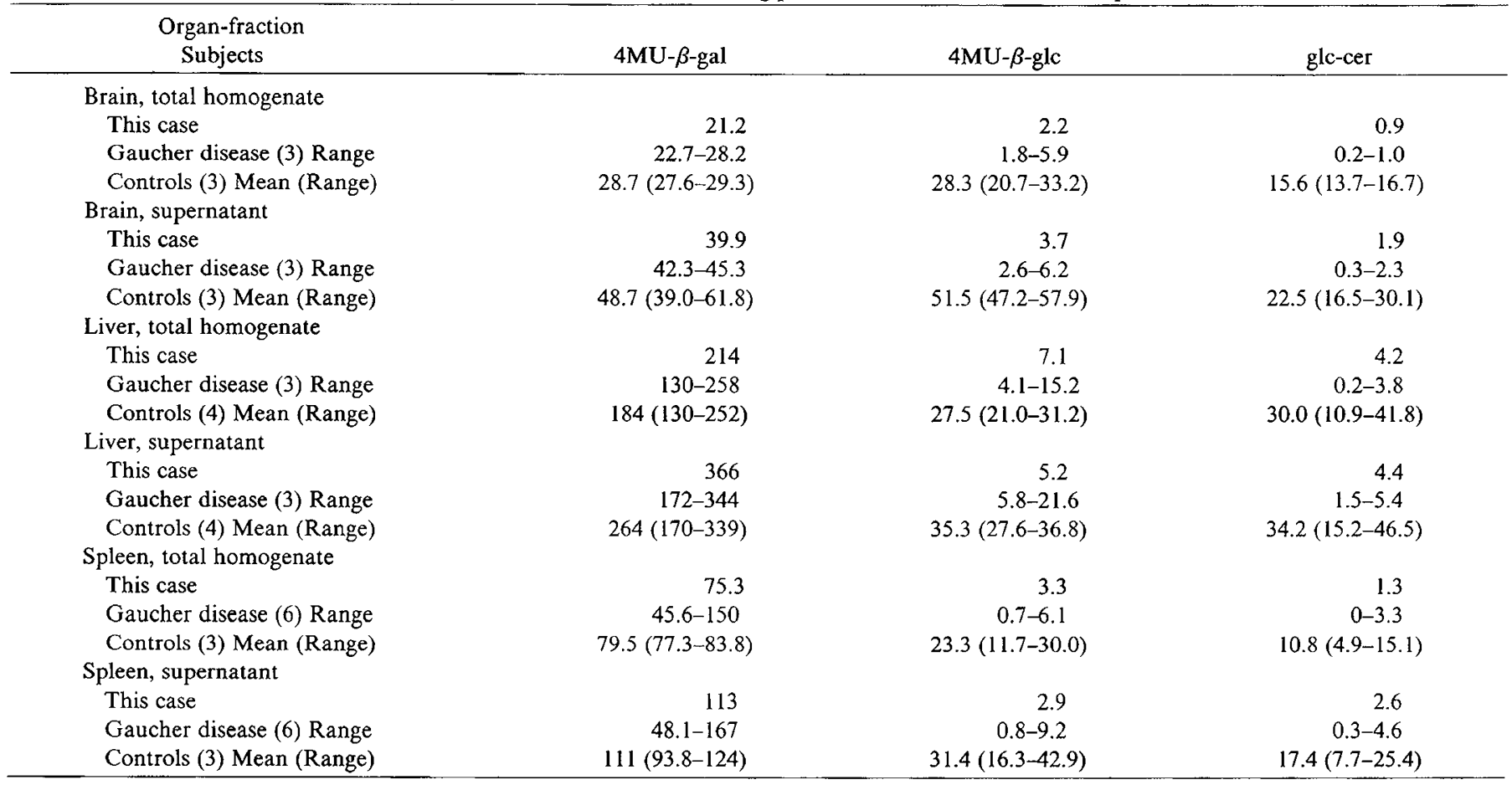

${ }^{1}$ Abbreviations, see "Abbreviations."

Table 4. Hybridization of cultured skin fibroblasts from glucocerebrosidase deficient patients ${ }^{1}$

\begin{tabular}{lll}
\hline \multicolumn{1}{c}{ Cell lines } & \multicolumn{1}{c}{$\begin{array}{c}\text { glc-cer } \\
\text { (nmole/mg pro- } \\
\text { tein/h) }\end{array}$} & $\begin{array}{c}\text { 4MU- } \beta \text {-glc } \\
\text { (nmole/mg pro- } \\
\text { tein } / \mathrm{h} \text { ) }\end{array}$ \\
\hline $\begin{array}{l}\text { Infantile GD } \times \text { Infantile GD } \\
\text { This case } \times \text { This case }\end{array}$ & 11.53 & 7.73 \\
Infantile GD $\times$ This case & 44.90 & 28.65 \\
& $35.48(28.22)^{2}$ & $16.65(18.19)^{2}$ \\
Adult GD $\times$ Adult GD & 53.34 & 20.21 \\
This case $\times$ This case & 51.25 & 37.86 \\
Adult GD $\times$ This case & $50.62(52.30)^{2}$ & $27.85(29.03)^{2}$ \\
& & \\
Adult GD $\times$ Adult GD & 42.04 & 21.06 \\
Infantile GD $\times$ Infantile GD & 10.42 & 10.72 \\
Adult GD $\times$ Infantile GD & $23.56(26.23)^{2}$ & $16.16(15.89)^{2}$ \\
\hline
\end{tabular}

\footnotetext{
'Abbreviations, see "Abbreviations." This table represents three separate experiments assayed in duplicate.

${ }^{2}$ Values in parentheses represent theoretical values.
}

Cell hybridization between cells from this patient and cells from adult and infantile Gaucher disease patients resulted only in the theoretical values (Table 4). The same occurred when cells from adult and infantile Gaucher patients were hybridized. This technique has been demonstrated to produce $50-70 \%$ multi-nucleated cells and clear evidence for complementation in some forms of $\beta$-galactosidase deficiency (18). The same result was found when the cells were cocultivated without the addition of polyethylene glycol. This could indicate that the mutations in these different clinical types of Gaucher disease are allelic or that the assay in mixtures of unhybridized and unselected hybrids is not sensitive enough to reveal complementation.

The spleen and liver were not enlarged when the child died at 12.5 years. Lipid analysis did not show the expected increase in glucosylceramide despite the low $\beta$-glucosidase values. The brain did shown an increase in glc-cer using a sensitive high performance liquid chromatographic technique. The level was lower than those found in brains from two acute neuropathic Gaucher disease patients. Our values for the glc-cer concentration in livers and spleens from Gaucher disease patients and controls are comparable to those previously reported from other laboratories (see reference 4 for a summary of values). Because this patient died at 12.5 years of age, it is possible that storage would have occurred if she had lived longer. Lipid analysis of spleen samples from presymptomatic patients with adult Gaucher disease are not available for comparison.

This manuscript describes a patient who has low $\beta$-glucosidase activity in all tissue samples examined, yet does not store glucosylceramide in the visceral organs. She appears to have had a neurologic disorder similar to patients with juvenile Gaucher disease. There have been patients with adult Gaucher disease who have neurologic signs and symptoms presenting later in life (9, 12); however, all of those patients have some evidence for splenomegaly and glc-cer accumulation. The father of this patient is the first cousin of a man who had a daughter with a more acute neuropathic form of Gaucher disease, and they both show carrier levels of $\beta$-glucosidase activity in their leukocytes (5). The mother of this patient has $\beta$-glucosidase activity in the normal range using the usual assay methods, but may have some decreased activity when assayed with an activator protein stimulated reaction. This child represents a case of subacute neuropathic Gaucher disease without storage of glucosylceramide in her liver or spleen. This is the reverse of most patients with Type 1 Gaucher disease who can have severe liver, spleen, and bone involvement but no obvious neurologic problems. This patient may provide us with important information on the role of $\beta$-glucosidase in the development of neurologic symptoms in Gaucher disease.

\section{REFERENCES AND NOTES}

1. Brady, R. O.: Glucosyl ceramide lipidosis: Gaucher's disease. In: J. B. Stanbury, J. B. Wyngaarden and D. S. Fredrickson. The Metabolic Basis of Inherited Disease, Fourth Edition, pp. 731-746 (McGraw-Hill, New York, 1978).

2. Davidson, R. L. and Gerald, P. S.: Improved techniques for the induction of mammalian cell hybridization by polyethylene glycol. Somatic Cell Genet., 2: 165 (1976).

3. Dreborg, S., Erikson, A. and Hagberg, B.: Gaucher disease-Norrbottnian Type I. General clinical description. Eur. J. Pediatr., 133: 107 (1980).

4. Fredrickson, D. S. and Sloan, H. R.: Glucosyl ceramide lipidosis: Gaucher's disease. In: J. B. Stanbury, J. B. Wyngaarden and D. S. Fredrickson. The 
Metabolic Basis of Inherited Disease, Third Edition, pp. 730-759 (McGraw Hill, New York, 1972).

5. Grover, W. D., Tucker, S. H. and Wenger, D. A.: Clinical variation in 2 related children with neuronopathic Gaucher disease. Ann. Neurol., 3: 281 (1978).

6. Herrlin, K. -M. and Hillborg, P. O.: Neurological signs in a juvenile form of Gaucher disease. Acta Paediatr. Scand., 5I: 137 (1962).

7. Lowry, O. H., Rosebrough, N. J., Farr, A. L., and Randall, R.: Protein measurement with Folin phenol reagent. J. Biol. Chem., 193: 265 (1951).

8. Maloney, A. F. J. and Cumings, J. N.: A case of juvenile Gaucher's disease with intraneuronal lipid storage. J. Neurol. Neurosurg. Psychiatry, 23: 207 (1960).

9. Miller, J. D., McCluer, R., and Kanfer, J. N.: Gaucher's disease: Neurologic disorder in adult siblings. Ann. Intern. Med., 78: 883 (1973).

10. Nishimura, R. N. and Barranger, J. A.: Neurologic complications of Gaucher's disease. Type 3. Arch. Neurol., 37: 92 (1980).

11. Nishimura, R., Omos-Lau, N., Ajmone-Marsan, C., and Barranger, J. A.: Electroencephalographic findings in Gaucher disease. Neurology, 30: 152 (1980).

12. Soffer, D., Yamanaka, T., Wenger, D. A., Suzuki, K., and Suzuki, K.: Central nervous system involvement in adult-onset Gaucher's disease. Acta Neuropathol., 49: 1 (1980).

13. Spiro, R. G.: Analysis of sugars found in glycoproteins. Methods Enzymol., VII: 3 (1966).

14. Stoffyn, P., Stoffyn, A., and Hauser G.: Structure of sulfatides biosynthesized in vitro. J. Lipid Res., 12: 318 (1971).

15. Ullman, M. D. and McCluer, R. H.: Quantitative microanalysis of perbenzoylated neutral glycosphingolipids by high-performance liquid chromatography with detection at $230 \mathrm{~nm}$. J. Lipid Res., 19: 910 (1978).

16. Wenger, D. A., Sattler, M., Clark, C., and Wharton, C.: I-Cell disease: activities of lysosomal enzymes toward natural and synthetic substrates. Life Sci., 19: 413 (1976).

17. Wenger, D. A., Clark, C., Sattler, M., and Wharton, C.: Synthetic substrate $\beta$ glucosidase activity in leukocytes: a reproducible method for the identification of patients and carriers of Gaucher disease. Clin. Genet., 13: 145 (1978).

18. Wenger, D. A., Sattler, M., Mueller, O. T., Myers, G., Schneiman, R. S., and Nixon, G. W.: Adult GMl gangliosidosis: Clinical and biochemical studies on two patients and comparison to other patients called variant or adult GMI gangliosidosis. Clin. Genet., 17: 323 (1980).

19. Wenger, D. A., Kudoh, T., Roth, S., and Sattler, M.: Sub-acute neuronopathic Gaucher disease without glucosylceramide storage. Clin. Res., 29: 135A (1981)

20. Wenger, D. A. and Olson, G. C.: Heterogeneity in Gaucher disease. In: J. W. Callahan and J. A. Lowden. Lysosomes and Lysosomal Storage Diseases. pp. 157-171 (Raven Press, New York, 1981).

21. Wenger, D. A. and Roth, S.: Homozygote and heterozygote identification. In: R. J. Desnick, S. Gatt and G. A. Grabowski. Gaucher Disease, A Century of Delineation and Research. pp. 551-572 (A. R. Liss, Inc., New York).

22. The authors thank Drs. Lucy Rorke and Robert E. Lee for their summaries of the pathologic findings and Harriet McKelvey for her excellent technical assistance with tissue culture.

23. Requests for reprints should be addressed to: Dr. David A. Wenger, Department of Pediatrics, Box C-233, University of Colorado Health Sciences Center, Denver, Colorado 80262.

24. This research was supported in part by grants from the National Institutes of Health (HD 08315, HD 10494 and NS 10698)

25. Received for publication July 1, 1982.

26. Accepted for publication October 15, 1982. 